\title{
Density waves in traffic flow
}

\author{
Takashi Nagatani \\ Division of Thermal Science, Department of Mechanical Engineering, Shizuoka University, Hamamatsu 432-8561, Japan
}

(Received 12 October 1999)

\begin{abstract}
Density waves are investigated in the car-following model analytically and numerically. This work is a continuation of our previous investigation of traffic flow in the metastable and unstable regions [Phys. Rev. E 58, 4271 (1998); 60, 180 (1999)]. The Burgers equation is derived for the density wave in the stable region of traffic flow by the use of nonlinear analysis. It is shown, numerically, that the triangular shock wave appears as the density wave at the late stage in the stable region. The decay rate of the shock wave is calculated and compared with the analytical result. It is shown that the density waves out of the coexisting curve, near the spinodal line, and within the spinodal line appear, respectively, as the triangular shock wave, the soliton, and the kink-antikink wave. The density waves are described, respectively, by the Burgers, Korteweg-de Vries, and modified Korteweg-de Vries equations.
\end{abstract}

PACS number(s): 05.90.+m, 47.35. $+\mathrm{i}, 89.40 .+\mathrm{k}$

\section{INTRODUCTION}

Recently, traffic problems have attracted considerable attention [1-3]. When car density is high in a highway, traffic jams occur and propagate as density waves [4-7]. The typical density wave has the kink-antikink form. It has been shown that the kink density wave is described by the modified Korteweg-de Vries (KdV) equation [8-11]. Also, the jamming transition between the freely moving traffic and jammed traffic has been described as a type of nonequilibrium phase transition [12-19]. Thus, the traffic flow has the two aspects of wave propagation and phase transition. Also, two-dimensional traffic models have been proposed for city traffic [20-23].

In modern traffic theory, it is well known that traffic jams occur in the high density region and propagate as kinkantikink density waves $[5,8-10]$. In the past, traffic jams have been treated as the soliton density wave [24,25] or triangular shock waves [26]. The nonlinear waves depend strongly on the traffic models [26]. Until now, it has been unclear as to whether or not the triangular shock wave occurs in modern traffic models. The car-following models with optimal velocity function are favorable among microscopic traffic models and have been studied in great detail by use of the numerical and analytical methods $[5,8,9,12]$.

Generally, traffic flow is divided into the three regions: the stable traffic region out of the coexisting curve, the metastability region between the coexisting and neutral stability lines, and the unstable region within the coexisting curve. Very recently, it has been shown that the kink density wave appears as the traffic jam in the unstable region and the soliton density wave occurs only near the neutral stability line in the car-following model [27]. However, the density waves in the stable region (or in the freely moving phase) have been little investigated in the car-following models. It is unknown how the density wave decays when a local density change occurs. The local density change is induced when a car decelerates to a low velocity or stops instantly. How does the local density variation relax to the uniform flow in the freely moving phase? In the relaxation process, where does the density wave appear? It is expected that the triangular shock wave appears as the density wave.

In this paper, we investigate the density wave appearing in the freely moving phase of the car-following models. We analyze the optimal velocity model by use of the nonlinear analysis method. We derive the Burgers equation to describe the density wave in the stable region. We show that there is a triangular shock wave described by the Burgers equation in the freely moving phase. We compare the numerical result with the analytical result. Finally, we find that the three types of nonlinear waves appear in the distinct three regions of traffic flow, and they are described by the Burgers, KdV, and modified $\mathrm{KdV}$ equations.

\section{MODELS AND BURGERS EQUATION}

We present the car-following models with the optimal velocity function [5,10,12]. Newell [24] and Whitham [25] have analyzed the traffic model described by the following equation of motion of car $j$ :

$$
\frac{d x_{j}(t+\tau)}{d t}=V\left(\Delta x_{j}(t)\right)
$$

where $x_{j}(t)$ is the position of car $j$ at time $t, \Delta x_{j}(t)$ $\left[=x_{j+1}(t)-x_{j}(t)\right]$ is the headway of car $j$ at time $t$, and $\tau$ is the delay time. The idea is that a driver adjusts the car velocity $d x_{j}(t) / d t$ according to the observed headway $\Delta x_{j}(t)$. The delay time $\tau$ allows for the time lag that it takes the car velocity to reach the optimal velocity $V\left(\Delta x_{j}(t)\right)$ when the traffic flow is varying.

By Taylor-expanding Eq. (1), one obtains the differential equation model [5]:

$$
\frac{d^{2} x_{j}(t)}{d t^{2}}=a\left[V\left(\Delta x_{j}(t)\right)-\frac{d x_{j}(t)}{d t}\right],
$$

where $a$ is the sensitivity of a driver [5] and $a=1 / \tau$.

Furthermore, by transforming time derivatives to asymmetric forward differences in Eq. (1), one can obtain the difference equation model [10]:

$$
x_{j}(t+2 \tau)=x_{j}(t+\tau)+\tau V\left(\Delta x_{j}(t)\right) .
$$




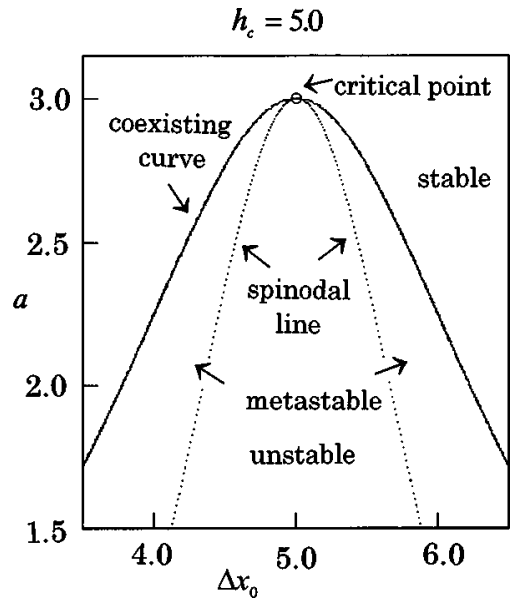

FIG. 1. Phase diagram of the difference equation model. There are the coexisting curve, the spinodal line, and the critical point similar to the conventional gas-liquid phase transition. Three regions in the traffic flow are distinguished: the stable region above the coexisting curve, the metastable region between the spinodal and coexisting curve, and the unstable region below the spinodal line.

Following asymmetric difference approximations are used to derive Eq. (3) from Eq. (2): $\tau^{2} d^{2} x_{j}(t) / d t^{2}=x_{j}(t+2 \tau)$ $-2 x_{j}(t+\tau)+x_{j}(t)$ and $\tau d x_{j}(t) / d t=x_{j}(t+\tau)-x_{j}(t)$. This asymmetry leads to the Burgers equations with different coefficients. The difference equation model is more suitable for computation since the time and space variables are discrete. It has been shown from simulation and analysis that the three models exhibit a similar traffic behavior and give similar phase diagrams $[8,10,11]$. The phase diagram of the difference equation model is shown in Fig. 1. In all three models, the traffic flow is divided into three regions: the first is the stable region above the coexisting curve, the second is the metastable region between the spinodal line and the coexisting curve, and the third is the unstable region below the spinodal line. In the unstable region, the traffic jam appears as the kink-antikink density wave. The kink jam has been presented by the solution of the modified $\mathrm{KdV}$ equation. Also, it has been shown that only near the neutral stability line (the spinodal line), does the soliton density wave appear [27]. The soliton density wave has been described by the $\mathrm{KdV}$ equation.

Generally, it is necessary that the optimal velocity function has the following properties: it is a monotonically increasing function and it has an upper bound (maximal velocity). The optimal velocity function has been given by

$$
V\left(\Delta x_{j}\right)=\frac{\nu_{\max }}{2}\left\{\tanh \left(\Delta x_{j}-h_{c}\right)+\tanh \left(h_{c}\right)\right\},
$$

where $h_{c}$ is the safety distance and $\nu_{\max }$ is the maximal velocity $[5,12]$. Equation (4) has the turning point (inflection point) at $\Delta x_{j}=h_{c}: V^{\prime \prime}\left(h_{c}\right)=\left.\left[d^{2} V\left(\Delta x_{j}\right) / d \Delta x_{j}^{2}\right]\right|_{\Delta x_{j}=h_{c}=0 .}$. It is important that the optimal velocity function has the turning point. Otherwise, one cannot have the kink-antikink density wave solution representing the traffic jam.

We call the three models described by Eqs. (1), (2), and (3) as models $A, B$, and $C$. We derive the Burgers equation for the density wave in the stable traffic flow region. The traffic flow is stable if the following condition is satisfied:

$$
\begin{gathered}
V^{\prime}\left(\Delta x_{0}\right)<\frac{1}{2 \tau} \text { for models } A \text { and } B, \\
V^{\prime}\left(\Delta x_{0}\right)<\frac{1}{3 \tau} \text { for model } C,
\end{gathered}
$$

where $\Delta x_{0}$ is the average headway and $V^{\prime}\left(\Delta x_{0}\right)$ $\left(=d V /\left.d \Delta x\right|_{\Delta x=\Delta x_{0}}\right)$ is the derivative of the optimal velocity function at $\Delta x=\Delta x_{0}$.

For later convenience, we rewrite Eqs. (1)-(3) as follows:

$$
\begin{gathered}
\frac{d x_{j}(t+\tau)}{d t}=V\left(\Delta x_{j+1}(t)\right)-V\left(\Delta x_{j}(t)\right), \\
\frac{d^{2} \Delta x_{j}(t)}{d t^{2}}=a\left\{V\left(\Delta x_{j+1}(t)\right)-V\left(\Delta x_{j}(t)\right)-\frac{d \Delta x_{j}}{d t}\right\}, \\
\Delta x_{j}(t+2 \tau)=\Delta x_{j}(t+\tau)+\tau\left\{V\left(\Delta x_{j+1}(t)\right)-V\left(\Delta x_{j}(t)\right)\right\} .
\end{gathered}
$$

We now consider long-wave modes in the stable traffic flow on coarse-grained scales. The simplest way to describe the long-wave modes is the long-wave expansion. We consider the slowly varying behavior at long wavelengths in the stable region. We extract slow scales for space variable $j$ and time variable $t$ [28]. For $0<\varepsilon \ll 1$, we therefore define slow variables $X$ and $T$ :

$$
X=\varepsilon(j+b t) \quad \text { and } \quad T=\varepsilon^{2} t,
$$

where $b$ is a constant determined later. We set the headway as

$$
\Delta x_{j}(t)=\Delta x_{0}+\varepsilon R(X, T) .
$$

By expanding Eqs. (7)-(9) to the third order of $\varepsilon$ with the use of Eqs. (10) and (11), one obtains the following nonlinear partial differential equations:

$$
\varepsilon^{2}\left(b-V^{\prime}\right) \partial_{x} R+\varepsilon^{3}\left[\partial_{T} R-V^{\prime \prime} R \partial_{x} R-\left(\frac{V^{\prime}}{2}-b^{2} \tau\right) \partial_{x}^{2} R\right]=0 .
$$

$$
\begin{gathered}
\varepsilon^{2}\left(a b-a V^{\prime}\right) \partial_{x} R+\varepsilon^{3}\left[a \partial_{T} R-a V^{\prime \prime} R \partial_{x} R-\left(\frac{a V^{\prime}}{2}-b^{2}\right) \partial_{x}^{2} R\right] \\
=0 . \\
\varepsilon^{2}\left(b-V^{\prime}\right) \tau \partial_{x} R+\varepsilon^{3}\left[\tau \partial_{T} R-V^{\prime \prime} \tau R \partial_{x} R\right. \\
\left.\quad-\left(\frac{V^{\prime} \tau}{2}-\frac{3 b^{2} \tau^{2}}{2}\right) \partial_{x}^{2} R\right] \\
=0
\end{gathered}
$$

where $\partial_{T}=\partial / \partial T, \partial_{x}=\partial / \partial X, \quad V^{\prime}=\left.[d V(\Delta x) / d \Delta x]\right|_{\Delta x=\Delta x_{0}}$, and $V^{\prime \prime}=\left.\left[d^{2} V(\Delta x) / d \Delta x^{2}\right]\right|_{\Delta x=\Delta x_{0}}$. Here we used the expansions shown in the Appendix. 
By taking $b=V^{\prime}$, the second order term of $\varepsilon$ is eliminated from Eqs. (12)-(14). One obtains the following partial differential equations:

$$
\begin{gathered}
\partial_{T} R-V^{\prime \prime} R \partial_{x} R=\left(\frac{V^{\prime}}{2}-V^{\prime 2} \tau\right) \partial_{x}^{2} R, \\
\partial_{T} R-V^{\prime \prime} R \partial_{x} R=\left(\frac{V^{\prime}}{2}-\frac{V^{\prime 2}}{a}\right) \partial_{x}^{2} R, \\
\partial_{T} R-V^{\prime \prime} R \partial_{x} R=\left(\frac{V^{\prime}}{2}-\frac{3 V^{\prime 2} \tau}{2}\right) \partial_{x}^{2} R .
\end{gathered}
$$

$V^{\prime \prime}$ is negative for $\Delta x>h_{c}$. The coefficients $\left(V^{\prime} / 2-V^{\prime 2} \tau\right)$, $\left(V^{\prime} / 2-V^{\prime 2} / a\right)$, and $\left(V^{\prime} / 2-3 V^{\prime 2} \tau / 2\right)$ of the second derivatives have positive values in the stable traffic region satisfying the stability conditions (5) and (6). Therefore, in the region of stable freely moving traffic flow, Eqs. (15)-(17) are just the Burgers equation. Equation (16) is consistent with Eq. (15) since $a=1 / \tau$. The solution of the Burgers equation for the asymptotic stage $(T \gg 1)$ is a train of $N$-shock waves [29]. The solution is given by

$$
\begin{aligned}
R(X, T)= & \frac{1}{\left|V^{\prime \prime}\right| T}\left[X-\frac{1}{2}\left(\eta_{j}+\eta_{j+1}\right)\right]-\frac{1}{2\left|V^{\prime \prime}\right| T} \\
& \times\left(\eta_{j+1}-\eta_{j}\right) \tanh \left[\frac{C_{1}}{4\left|V^{\prime \prime}\right| T}\right. \\
& \left.\times\left(\eta_{j+1}-\eta_{j}\right)\left(X-\xi_{j}\right)\right]
\end{aligned}
$$

where $C_{1}=V^{\prime} / 2-V^{\prime 2} \tau$ for Eqs. (15) and (16), $C_{1}=V^{\prime} / 2$ $-3 V^{\prime 2} \tau / 2$ for Eq. (17). The coordinates of the shock fronts are given by $\xi_{j}(j=1,2, \ldots, N)$ and those of the intersections of the slopes with the $x$ axis by $\eta_{j}(j=1,2, \ldots, N)$.

Since the space variable $X$ is given by Eq. (10), the triangular shock wave propagates backward with the propagation velocity $b$. The propagation velocity $\nu_{p}$ is given by

$$
\nu_{p}=V^{\prime}\left(\Delta x_{0}\right)
$$

The shock wave propagates backwards only with respect to the moving vehicles (i.e., relative to the index $j$ ), but the shock propagates forward in the absolute system, if one is in the region of the free traffic. The propagation velocity decreases with increasing the average headway. The propagation velocity does not depend on the sensitivity. In contrast, the propagation velocity of the kink density does not depend on the average density but on the sensitivity $[8,27]$.

\section{SIMULATION}

We carry out simulation to compare the simulation result with the analytical result. The investigated system is a difference equation model (3) since it is more suitable for computation. In the nonlinear analysis, we obtain the shock wave solution as the density wave appearing in the stable traffic flow region. However, a priori it could not be assumed that the car-following models would yield the triangular shock waves. Therefore, simulation is carried out to validate two points: (1) First it has to be shown that the triangular shock
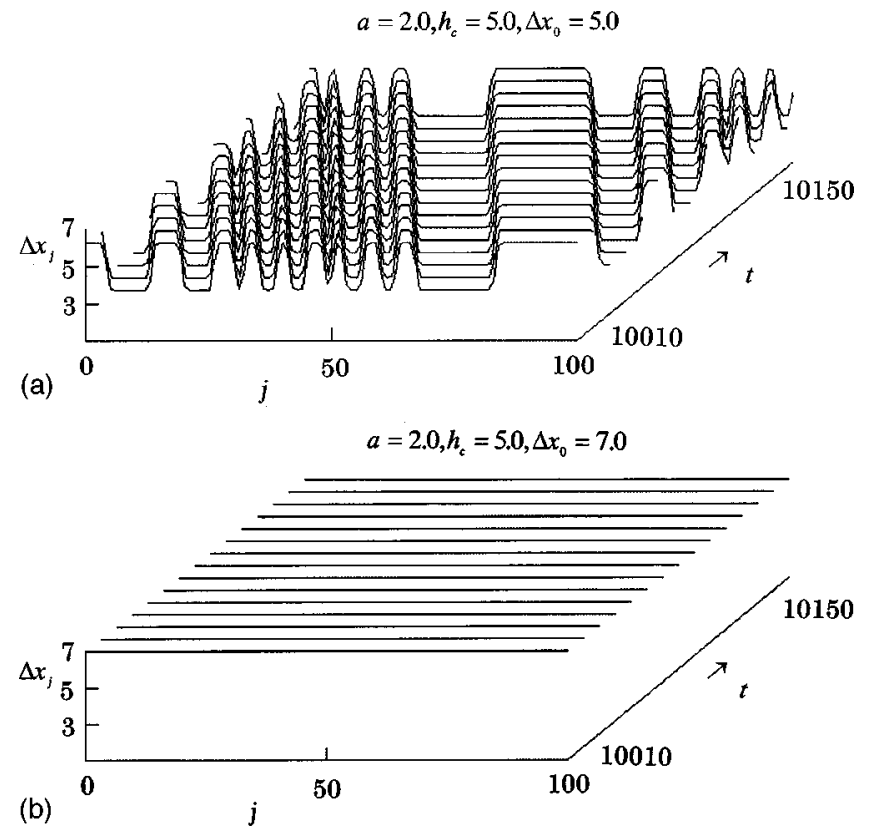

FIG. 2. Space-time evolution of the headway after a sufficiently large time. (a) Pattern for the coexisting phase. (b) Pattern for the freely moving phase.

waves indeed appear in the stable region of the car-following model except for the kink density wave; (2) next the applicability of the nonlinear analysis has to be proven.

The boundary is periodic. We study the space-time evolution of the headway for various values of sensitivity $a$. As a result, three types of traffic flow are distinguished: (1) a freely moving phase, (2) a coexisting phase in which the kink-antikink density wave appears, and (3) a uniformly congested phase. Figure 2 shows the typical traffic patterns. The pattern in Fig. 2(a) exhibits the space-time evolution of the headway for the coexisting phase after a sufficiently large time. The kink-antikink density wave appears as the traffic jam. The pattern in Fig. 2(b) exhibits the space-time evolution of the headway for the freely moving phase after a sufficiently large time. The uniform traffic flow with low density appears. Any initial disturbances decay and any initial traffic flow with a nonuniform density profile evolves to the uniformly traffic flow.

Until now, the coexisting phase has been investigated in detail. Here, we focus our attention to the freely moving

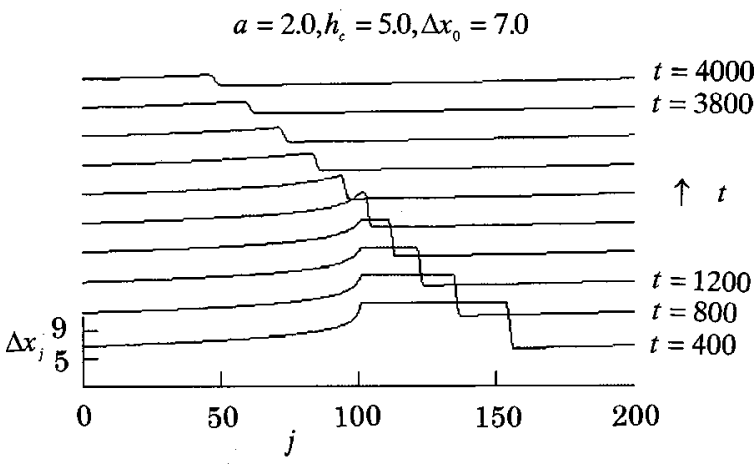

FIG. 3. Time evolution of the headway profiles when the initial density profile has the kink-antikink form in the freely moving phase. 


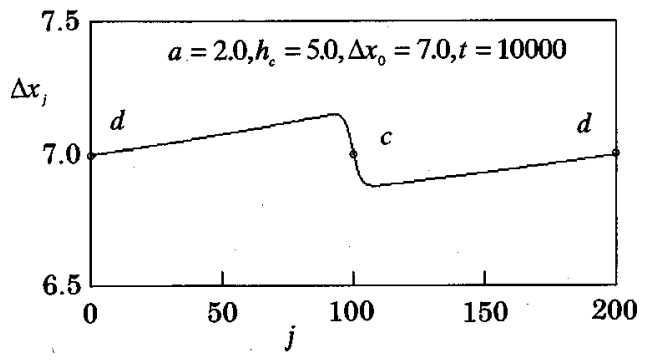

FIG. 4. Headway profile of the triangular shock wave obtained at $t=10000$. Points $c$ and $d$ indicate, respectively, the positions of the shock front and the intersection of the slope.

phase. We consider the relaxation process of the nonuniform flow to the uniform steady flow. We study how the initial density profile with kink-antikink form evolves to the uniform traffic flow. The initial headway profile is chosen as follows:

$$
\begin{aligned}
& \Delta x_{j}(0)=\Delta x_{j}(1)=\Delta x_{0}-2.0 \quad \text { for } 1 \leqslant j \leqslant N / 2, \\
& \Delta x_{j}(0)=\Delta x_{j}(1)=\Delta x_{0}+2.0 \quad \text { for } \quad N / 2<j \leqslant N,
\end{aligned}
$$

where $\Delta x_{0}$ is the average headway. Figure 3 shows the time evolution of the headway profiles at $t=400,800,1200, \ldots$, 3800,4000 where $a=2.0, \Delta x_{0}=7.0, h_{c}=5.0, N=200$. The initial kink-antikink headway profile decays through the triangular shock wave to the uniform flow. Figure 4 shows the headway profile obtained at $t=10000$. The point $c$ indicates the position of the intersection of the shock front with $\Delta x_{j}(10000)=\Delta x_{0}=7.0$. The point $d$ indicates the position of the intersection of the slope with $\Delta x_{j}(10000)=\Delta x_{0}$ $=7.0$ line. The headway profile obtained from simulation agrees with the triangular shock wave solution (18). We find that the initial kink-antikink headway profile evolves to the triangular shock wave at an asymptotic stage. Furthermore, we study the decay rate of the slopes of the triangular shock wave in order to prove the consistency with the headway profile (18). The triangular shock wave has the two intersections of the shock front and the triangular part with $\Delta x_{j}(10000)=\Delta x_{0}=7.0$ line. From Eq. (18), the two slopes at the intersections scale, respectively, as

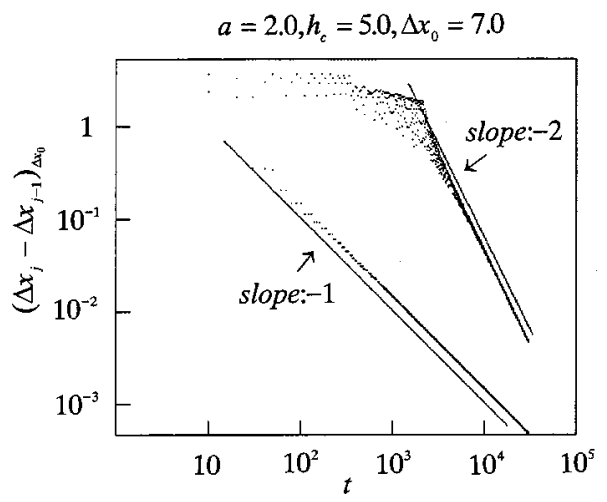

FIG. 5. Log-log plot of the slopes of the triangular part and shock front against time $t$ where $a=2.0, \Delta x_{0}=7.0, h_{c}=5.0$. The two straight lines with slopes -1 and -2 are shown by the solid lines near the simulation data.

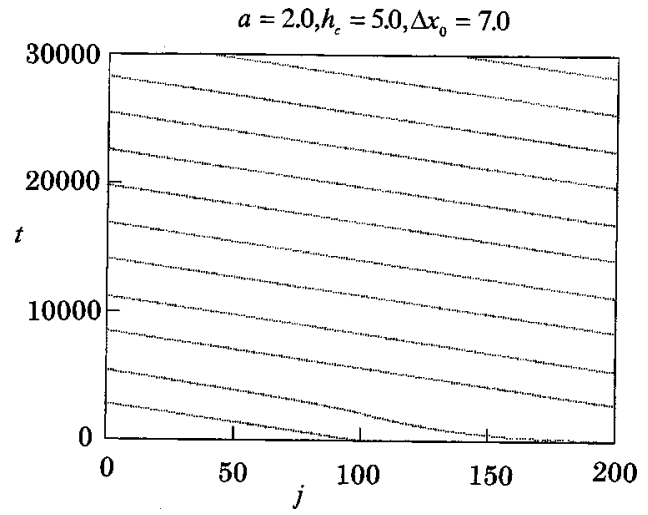

FIG. 6. Plot of the two intersections $c$ and $d$ (see Fig. 4) of the triangular shock wave against $t$ until $t=30000$ where $a=2.0$, $\Delta x_{0}=7.0, h_{c}=5.0, N=200$.

$$
\left(\Delta x_{j}-\Delta x_{j-1}\right)_{\Delta x_{0}} \approx t^{-1} \text { at point } d
$$

(the slope of the triangular part),

$\left(\Delta x_{j}-\Delta x_{j-1}\right)_{\Delta x_{0}} \approx t^{-2}$ at point $c$

(the slope of the shock front).

Figure 5 shows the log-log plot of the slopes against time $t$ until time $t=30000$ where $a=2.0, \Delta x_{0}=7.0, h_{c}=5.0, N$ $=200$. The two solid lines are shown near the simulation data. They indicate, respectively, the straight lines with the slopes -1 and -2 . We find that the slopes decays as Eqs. (21) and (22) with the scaling exponents -1 and -2 .

We study the propagation velocity of the triangular shock wave. Figure 6 shows the plot of the two intersections $c$ and $d$ (see Fig. 4) of the triangular shock wave with respect to time $t$ until time $t=30000$ where $a=2.0, \Delta x_{0}=7.0, h_{c}$ $=5.0, N=200$. Except for an initial stage, the intersections propagate with the constant velocity. The propagation velocity $\nu_{p}$ is given by the slope of the locus. The propagation velocity depends highly on the average density (or average headway). Figure 7 shows the plot of the propagation velocity $\nu_{p}$ against the average headway $\Delta x_{0}$ where $a=2.0, h_{c}$ $=5.0$. The circular points indicate the simulation result. The propagation velocity decreases with increasing the average headway. The solid line represents the analytical result $\nu_{p}$

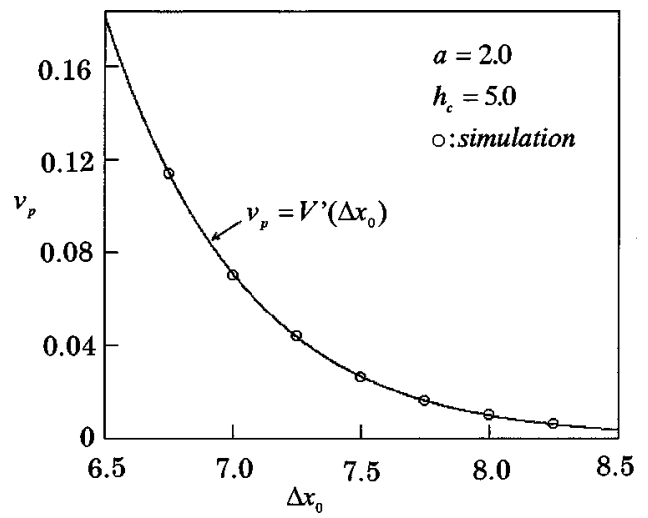

FIG. 7. Plot of the propagation velocity $\nu_{p}$ against the average headway $\Delta x_{0}$ where $a=2.0, h_{c}=5.0$. 


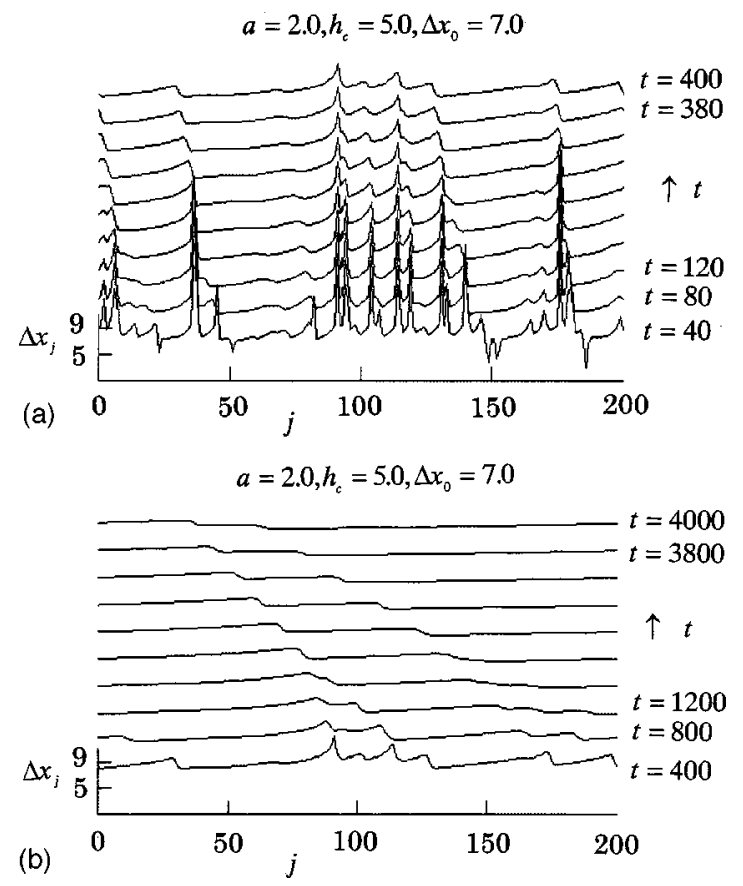

FIG. 8. Time evolution of the headway profiles for the initial random headway profile at (a) $t=40,80,120, \ldots, 380,400$, (b) $t$ $=400,800,1200, \ldots, 3800,4000$ where $a=2.0, \Delta x_{0}=7.0, h_{c}$ $=5.0, N=200$.

$=V^{\prime}\left(\Delta x_{0}\right)$. The simulation result is consistent with the analytical result. The propagation velocity does not depend on the sensitivity.

We study the relaxation process of the random headway profile to the uniform steady flow. The initial random headway profile is chosen as follows:

$$
\Delta x_{j}(0)=\Delta x_{j}(1)=7.0-10.0\left(R_{j}-0.5\right),
$$

where $R_{j}$ is a real number between zero and unity generated by random number generator at car $j$. Figure 8 shows the time evolution of the headway profiles at (a) $t=40,80,120$, ..., 380, 400, (b) $t=400,800,1200, \ldots, 3800,4000$ where $a=2.0, \Delta x_{0}=7.0, h_{c}=5.0, N=200$. The initial irregular headway profile decays through the triangular shock waves to the uniform flow. Thus, we find that the nonuniform density profile evolves through the triangular shock waves to the uniform flow in the stable traffic flow region.

\section{NONLINEAR WAVES}

For comparison, we shortly repeat the derivations of the modified KdV equation of the kink density wave and the $\mathrm{KdV}$ equation of the soliton density wave $[8,12,27]$. The kink density wave appears below the coexisting curve. The soliton density wave appears near the spinodal line (see Fig. 1). The kink density wave is robust. Even if any disturbances are added to the kink density wave, the deformed density wave returns to the kink density wave in the course of time. On the other hand, the soliton density wave is unstable even if perturbations are very small. All perturbations will either develop to the kink density wave or dissolve in the course of time.

The modified $\mathrm{KdV}$ equations are derived in the unstable region just below the critical point $[8,12]$. The small positive scaling parameter $\varepsilon\left(=\sqrt{\left(a_{c} / a\right)-1}\right) \ll 1$ is introduced. It represents the deviation from the critical point $a_{c}$. One defines the slow variables $X$ and $T$ :

$$
X=2 \varepsilon\left(j+V^{\prime} t\right) \quad \text { and } \quad T=\frac{4}{3} \varepsilon^{3} V^{\prime} t,
$$

where $V^{\prime}$ is the derivative at the critical point: $V^{\prime}$ $=\left.[d V(\Delta x) / d \Delta x]\right|_{\Delta x=h_{c}}$. The headway is set as follows:

$$
\Delta x_{j}(t)=h_{c}+2 \varepsilon \sqrt{\frac{V^{\prime}}{\left|V^{\prime \prime \prime}\right|}} R(X, T) .
$$

where $V^{\prime \prime \prime}=\left.\left[d^{3} V(\Delta x) / d \Delta x^{3}\right]\right|_{\Delta x=h_{c}}$. By inserting Eq. (25) into Eq. (8) and expanding to the fifth order of $\varepsilon$, one obtains the modified $\mathrm{KdV}$ equation with the perturbed term:

$$
\varepsilon^{4}\left(\partial_{T} R-\partial_{x}^{3} R+\partial_{x} R^{3}\right)+\frac{3}{2} \varepsilon^{5}\left(\partial_{x}^{2} R+\partial_{x}^{4} R-\frac{2}{3} \partial_{x}^{2} R^{3}\right)=0 .
$$

Finally, one obtains the kink solution from Eq. (26):

$$
\begin{aligned}
\Delta x_{j}(t)= & h_{c} \pm \sqrt{\frac{5 V^{\prime}\left(h_{c}\right)\left(\frac{a_{c}}{a}-1\right)}{\left|V^{\prime \prime \prime}\left(h_{c}\right)\right|}} \\
& \times \tanh \left[\sqrt{\frac{5\left(\frac{a_{c}}{a}-1\right)}{2}}\right. \\
& \left.\times\left\{j+\left(1-\frac{5\left(\frac{a_{c}}{a}-1\right)}{6}\right) V^{\prime}\left(h_{c}\right) t\right\}\right] .
\end{aligned}
$$

The modified $\mathrm{KdV}$ equation is derived just below the critical point. However, the kink density wave solution (27) agrees with the simulation result far from the critical point $[8,12]$.

We derive the $\mathrm{KdV}$ equation from Eq. (2). The $\mathrm{KdV}$ equations are derived near the spinodal line (the neutral stability line) [27]. The small positive scaling parameter $\varepsilon$ $\left(=\sqrt{\left|a_{s} / a-1\right|}\right) \ll 1$ is introduced. It represents the derivation from the neutral stability line $a_{s}\left[=2 V^{\prime}\left(\Delta x_{0}\right)\right]$. One defines the slow variables $X$ and $T$ :

$$
X=\varepsilon\left(j+V^{\prime} t\right) \text { and } T=\varepsilon^{3} t,
$$

where $V^{\prime}$ is the derivative on the neutral stability line: $V^{\prime}$ $=\left.[d V(\Delta x) / d \Delta x]\right|_{\Delta x=\Delta x_{0}}$. The headway is set as follows:

$$
\Delta x_{j}(t)=\Delta x_{0}+\varepsilon^{2} R(X, T) .
$$

By inserting Eq. (29) into Eq. (8) and expanding to the sixth order of $\varepsilon$, one obtains the $\mathrm{KdV}$ equation with the perturbed term: 


$$
\begin{aligned}
& \varepsilon^{5} a\left(\partial_{T} R-\frac{V^{\prime}}{6} \partial_{x}^{3} R-V^{\prime \prime} R \partial_{x} R\right) \\
& +\varepsilon^{6}\left[\frac{a V^{\prime}}{2} \partial_{x}^{2} R+\left(\frac{V^{\prime 2}}{3}-\frac{a V^{\prime}}{24}\right) \partial_{x}^{4} R+\left(V^{\prime} V^{\prime \prime}\right.\right. \\
& \left.\left.-\frac{a V^{\prime \prime}}{4}\right) \partial_{x}^{2} R^{2}\right]=0 .
\end{aligned}
$$

Finally, one obtains the soliton solution from Eq. (30):

$$
\begin{aligned}
\Delta x_{j}(t)= & \Delta x_{0}+\frac{14 V^{\prime}}{3 V^{\prime \prime}}\left|\frac{a_{s}}{a}-1\right| \\
& \times \operatorname{sech}^{2} \mid \sqrt{\frac{\mid a_{s}}{a}-1 \mid} \\
& \left.\times\left\{j+\left(1+\frac{14\left|\frac{a_{s}}{a}-1\right|}{9}\right) V^{\prime} t\right\}\right] .
\end{aligned}
$$

The KdV equation is derived just near the spinodal line.

Thus, the kink and soliton density waves are observed, respectively, below the coexisting curve and near the spinodal line. On the other hand, the triangular shock wave is observed above the coexisting curve when the uniform traffic flow is deformed by the disturbances. The three types of density waves appear corresponding to the three distinct regions of traffic flow. Here, the three types of density waves have been found for the same (optimal velocity) model, with just different parameters.

\section{SUMMARY}

We have studied the relaxation process of nonuniform density profile in the stable traffic flow to the uniform density profile with the use of the car-following models. We have shown, analytically and numerically, how the initially nonuniform density profile evolves to the uniform density profile in the stable traffic region. We have found that the nonuniform density profile relaxes through the triangular shock wave to the uniform density profile. It has been shown that the triangular shock wave is described by the Burgers equations. We have found that the analytical solution of the Burgers equation is consistent with the triangular shock wave obtained by simulation. Finally, we have concluded that the three types of density waves appear in the distinct three regions of traffic flow: the first is the kink-antikink density wave appearing in the unstable region within the spinodal line, the second is the soliton density wave appearing near the spinodal line, and the third is the triangular shock wave appearing in the stable region out of the coexisting curve. These density waves are described, respectively, by the modified $\mathrm{KdV}$ equation, the $\mathrm{KdV}$ equation, and the Burgers equation.

In past works, the traffic jam has been described by the Burgers equation in a particular hydrodynamic model. However, the triangular density wave found in this paper is to the best of our knowledge, definitely different from any in past works since the triangular shock wave is one of the three types of density waves and the Burgers equation is derived from the microscopic model.

\section{APPENDIX}

In this appendix, we present the expansions of each term in Eqs. (7)-(9) to third order of $\varepsilon$ :

$$
\begin{aligned}
& \frac{d \Delta x_{j}(t)}{d t}=\varepsilon^{2} b \partial_{x} R+\varepsilon^{3} \partial_{T} R \\
& \frac{d^{2} \Delta x_{j}(t)}{d t^{2}}=\varepsilon^{3} b^{2} \partial_{x}^{2} R \\
& \frac{d \Delta x_{j}(t+\tau)}{d t}=\varepsilon^{2} b \partial_{x} R+\varepsilon^{3} b^{2} \tau \partial_{x}^{2} R+\varepsilon^{3} \partial_{T} R, \\
& \Delta x_{j}(t+\tau)=\Delta x_{0}+\varepsilon R+\varepsilon^{2} b \tau \partial_{x} R+\varepsilon^{3} \frac{(b \tau)^{2}}{2} \partial_{x}^{2} R+\varepsilon^{3} \tau \partial_{T} R, \\
& \Delta x_{j}(t+\tau)=\Delta x_{0}+\varepsilon R+\varepsilon^{2} 2 b \tau \partial_{x} R+\varepsilon^{3} \frac{4(b \tau)^{2}}{2} \partial_{x}^{2} R \\
& +\varepsilon^{3} 2 \tau \partial_{T} R \\
& \Delta x_{j+1}(t)=\Delta x_{0}+\varepsilon R+\varepsilon^{2} \partial_{x} R+\frac{\varepsilon^{3}}{2} \partial_{x}^{2} R, \\
& V\left(\Delta x_{j}\right)=V\left(\Delta x_{0}\right)+V^{\prime}\left(\Delta x_{0}\right)\left(\Delta x_{j}-\Delta x_{0}\right) \\
& +\frac{V^{\prime \prime}\left(\Delta x_{0}\right)}{2}\left(\Delta x_{j}-\Delta x_{0}\right)^{2}, \\
& V\left(\Delta x_{j+1}\right)-V\left(\Delta x_{j}\right)=\varepsilon^{2} V^{\prime} \partial_{x} R+\varepsilon^{3}\left(V^{\prime \prime} R \partial_{x} R+\frac{V^{\prime}}{2} \partial_{x}^{2} R\right) .
\end{aligned}
$$

By inserting (A1)-(A8) into Eqs. (7)-(9), one obtains Eqs. (12)-(14).
[1] Traffic and Granular Flow, edited by D. E. Wolf, M. Schreckenberg, and A. Bachem (World Scientific, Singapore, 1996).

[2] D. Helbing, Verkehrsdynamik (Springer, Berlin, 1997).

[3] Traffic and Granular Flow 97, edited by M. Schreckenberg and D. E. Wolf (Springer, Singapore, 1998).

[4] K. Nagel and M. Schreckenberg, J. Phys. I 2, 2221 (1992).

[5] M. Bando, K. Hasebe, A. Nakayama, A. Shibata, and Y. Sug- iyama, Phys. Rev. E 51, 1035 (1995).

[6] B. S. Kerner, P. Konhauser, and M. Schilke, Phys. Rev. E 51, 6243 (1995).

[7] B. S. Kerner and H. Rehborn, Phys. Rev. E 53, R1297 (1996).

[8] T. Komatsu and S. Sasa, Phys. Rev. E 52, 5574 (1995).

[9] T. Nagatani and K. Nakanishi, Phys. Rev. E 57, 6415 (1998).

[10] T. Nagatani, K. Nakanishi, and H. Emmerich, J. Phys. A 31, 
5431 (1998).

[11] T. Nagatani, Physica A 261, 599 (1998).

[12] T. Nagatani, Phys. Rev. E 58, 4271 (1998).

[13] S. Krauss, P. Wagner, and C. Gawron, Phys. Rev. E 55, 5597 (1997).

[14] K. Nagel, D. E. Wolf, P. Wagner, and P. Simon, Phys. Rev. E 58, 1425 (1998).

[15] D. Helbing and M. Schreckenberg, Phys. Rev. E 59, R2505 (1999).

[16] D. Helbing, Phys. Rev. E 53, 2366 (1996).

[17] M. Treiber, A. Hennecke, and D. Helbing, Phys. Rev. E 59, 239 (1999).

[18] H. Y. Lee, H. W. Lee, and D. Kim, Phys. Rev. E 59, 5101 (1999).

[19] D. Helbing, A. Hennecke, and M. Treiber, Phys. Rev. Lett. 82,
4360 (1999).

[20] O. Biham, A. A. Middleton, and D. A. Levine, Phys. Rev. A 46, R6124 (1992).

[21] T. Nagatani, Phys. Rev. E 48, 3290 (1993).

[22] J. A. Cuesta, F. C. Matinez, J. M. Nolera, and A. Sanchez, Phys. Rev. E 48, 4175 (1993).

[23] T. Nagatani, Phys. Rev. E 59, 4857 (1999).

[24] G. F. Newell, Oper. Res. 9, 209 (1961).

[25] G. B. Whitham, Proc. R. Soc. London, Ser. A 428, 49 (1990). [26] K. Nagel, Phys. Rev. E 53, 4655 (1996).

[27] M. Muramatsu and T. Nagatani, Phys. Rev. E 60, 180 (1999).

[28] M. C. Cross and P. C. Hohenberg, Rev. Mod. Phys. 65, 851 (1993).

[29] T. Tatsumi and S. Kida, J. Fluid Mech. 55, 659 (1972). 